\title{
AMENDMENTS
}

\section{Author Correction: Sequence-defined multifunctional polyethers via liquid-phase synthesis with molecular sieving}

Ruijiao Dong (D), Ruiyi Liu, Piers R. J. Gaffney, Marc Schaepertoens, Patrizia Marchetti, Christopher M. Williams, Rongjun Chen $\mathbb{D}$ and Andrew G. Livingston (D)

Correction to: Nature Chemistry https://doi.org/10.1038/s41557-018-0169-6, published online 3 December 2018.

In the version of this Article originally published, the authors inadvertently cited ref. 10 in two places in the first paragraph. They would like to clarify that it should not have been cited in the sentence that starts "Polymer chemists have employed strategies such as single monomer insertion..." as it mistakenly implied that the IEG+ method described in ref. 10 could not produce unimolecular polymers; it can do so, as was demonstrated in ref. 10. The authors would also like to clarify that ref. 10 should not have been cited in the sentence that starts "Moreover, solid-phase synthesis is generally difficult to scale up..., as it implied that ref. 10 uses solid-phase synthesis; it does not, and is a purely liquid-phase process. The citation of ref. 10 has now been removed from these two sentences, but has been included elsewhere in the first two paragraphs of the Article as follows. In the first paragraph, at the end of the sentence "In iterative synthesis, specific monomers are added one at a time, or as multiples, to the end of a growing polymer chain, then reaction debris is separated from the chain extended polymer, and the cycle is repeated using the next monomer in the sequence ${ }^{10-12}$."; this sentence has been further amended to indicate multiple monomers can also be added. The reference has also been added to the end of the first sentence of the second paragraph, which starts "Consequently, liquid-phase iterative synthetic methods..., and in the third sentence of that paragraph, which now starts "For example, Johnson ${ }^{10}$, Whiting...."

Published online: 8 January 2019

https://doi.org/10.1038/s41557-019-0212-2

\section{Publisher Correction: Ephemeral elements}

Michelle Francl

Correction to: Nature Chemistry https://doi.org/10.1038/s41557-018-0189-2, published online 14 December 2018.

In the version of this Comment originally published, the name of the author of ref. 10 was incorrectly given as "Chapman, J."; this has now been corrected to "Chapman, K.".

Published online: 8 January 2019

https://doi.org/10.1038/s41557-019-0213-1

\section{Publisher Correction: Neutron stardust and the elements of Earth}

\section{Brett F. Thornton and Shawn C. Burdette}

Correction to: Nature Chemistry https://doi.org/10.1038/s41557-018-0190-9, published online 14 December 2018.

In the version of this Comment originally published, the image was incorrectly credited to Chelsea Anne Bar; it should have been to Brett F. Thornton. This has now been corrected.

Published online: 10 January 2019

https://doi.org/10.1038/s41557-019-0214-0 